\title{
Linking PCNA-dependent replication and ATR by human Claspin
}

Jean-Marc Brondello ${ }^{1^{*}}$, Bernard Ducommun ${ }^{2}$, Anne Fernandez ${ }^{3}$ and Ned J. Lamb ${ }^{3}$

(1) INSERM EMI 0229 Génotypes et Phénotypes Tumoraux CRLC Val d'Aurelle 34298 Montpellier cedex 5

(2)LBCMCP - CNRS UMR5088 - IFR 109 Université P. Sabatier, 118 route de Narbonne, 31062 Toulouse Cedex FRANCE (France).

(3) Cell Biology Unit, Institut de génétique Humaine, CNRS UPR 1142, 141 rue de la Cardonille, 34396 Montpellier cedex 5 (France)

Running title: DNA damage checkpoint, ATR, Claspin, PCNA, replication

*To whom correspondance should be addressed

(Tel: 334676137 67, Fax: 334676130 41, Email: jmbrondello@valdorel.fnclcc.fr) 


\begin{abstract}
Recent studies in Xenopus have identified a new checkpoint protein called Claspin that is believed to transduce the checkpoint DNA damage signals to Chk1 kinase. Here we show that the human Claspin homolog is a chromatin bound protein either in absence or in the presence of damaged DNA, independently of its association with ATR. Furthermore, we show that human Claspin is found in complex with PCNA, an essential component of the DNA replication machinery, and is released upon DNA replication arrest. Interfering with PCNA function by overexpression of p21 mutant, impaired in its interaction with Cdks but not with PCNA, leads to ATR-dependent Chk1 activation. These findings suggest that the dissociation of Claspin-PCNA could be part of the signal leading to Chk1 activation.
\end{abstract}




\section{INTRODUCTION}

Entry into mitosis $(\mathrm{M})$ is actively coupled to the completion of DNA synthesis (S) through the intermediate of Checkpoints. These mechanisms, conserved from yeast to humans and known as DNA structure checkpoints, are survey pathways that delay cell cycle progression to allow DNA repair in response to any type of DNA damage. Thus, defects in these checkpoint functions can result in genetic instability and ultimately cancer.

Genetic studies in yeast and metazoans uncovered a group of proteins required for the DNA structure checkpoint. Thus, two PI-3-like kinase family members, ATM (ataxia telangiectasia $[\mathrm{AT}]$ mutated) and ATR (ATM and $\operatorname{rad} 3$ related), are essential checkpoint transducers. Cells from AT patients are hypersensitive to DNA damage involving double strand breaks such as those induced by ionizing radiation or radiomimetic agents like bleomycin (BL). On the other hand, ATR seems to be involved mainly in the response to DNA replication blocks caused by Hydroxyurea (HU) or UV-induced DNA damage. ATM and ATR associated with several others sensors checkpoint proteins transmit DNA damage signals to at least two effector kinases, Chk1 and Chk2 [22]. These kinases regulate proteins that control Cdks, the cyclindependent kinases that catalyze specific cell cycle events. Both checkpoint kinases are required to increase the abundance of $\mathrm{p} 53$, a tumor suppressor protein that performs inhibitory functions by transcriptionally inducing $\mathrm{p} 21$, a cyclin-dependent kinase inhibitor. In addition, Chk1 and 
Chk2 phosphorylate, at least in vitro, and thereby inhibit Cdc25A, B and C, the dual specificity phosphatases that activate Cdks. In response to HU or UV, Chk1 is activated by phosphorylation on at least two residues, Ser317 and Ser345 in an ATR-dependent manner [1], while Chk2 is phosphorylated on Thr68 residue in response to double strand breaks in an ATM-dependent manner [2].

Recent work has identified a new protein, Claspin, involved in ATR-dependent activation of Chk1. In Xenopus extracts and human cells, Claspin interacts weakly with the activated /phosphorylated form of Chk1 in response to DNA replication arrest [3] [4]. Moreover, immunodepletion of Claspin from frog egg extracts or Claspin gene ablation by expression smallinterfering RNA in human cells, leads to a DNA replication checkpoint deficiency without detectable Chk1 activation/ or phosphorylation [3] [4]. Although Xenopus Claspin is believed to be a chaperone protein allowing Chk1 phosphorylation [5], its role as part of the sensor of DNA replication block remains under investigation.

Here we show that human Claspin protein is an chromatin bound protein. We found that a fraction of human Claspin interacts with ATR and with PCNA, a essential DNA replication protein associated with DNA polymerase delta and epsilon. Interestingly, these two interactions are released upon DNA damage although Claspin remains associated with DNA. Finally, we show that functionnal knock out of PCNA leads to ATR-dependent Chk1 activation. Taken together, these data support that Claspin protein is an adapator protein binding ATR and involved as link between DNA structure checkpoint components and the replication machinery itself. 


\section{Results}

Chromatin association of human claspin independently of DNA damage

In order to follow human Claspin protein expression, we raised rabbit polyclonal antibodies (CLN\#24) against a 20 amino acid peptide (AA16-36). Affinity purified serum was tested by western blot on asynchronously growing Hela cell extracts. As shown in figure 1A, CLN\#24 recognizes one major band migrating around $170 \mathrm{kDa}$, which was competed by the immunogenic peptide, and gives a pattern identical to a commercially available antibody directed against the C-terminal region of human Claspin protein (Figure 1A). Unfortunatly, none of these available antibodies were able to immunoprecipitate human Claspin from cell extracts.

In Xenopus extracts, Claspin protein has been found associated with chromatin [6]. To determine if this was also the case for human Claspin, we used cell fractionation approaches followed by western blotting. Human Claspin is barely detectable in the detergent soluble fraction of proliferating Hela extracts, while more than $90 \%$ of the protein is present in the Triton insoluble fraction (Figure. 1B). As fractionation controls, we used a cytosolic kinase, PRK [7] and a partialy chromatin bound protein, MCM2, involved in DNA replication process. As shown in Figure 1B, PRK is present in the soluble fraction whereas MCM2 is found in both insoluble and soluble fractions, as described previously [8]. As expected for a chromatin bound protein, human Claspin was released from its Triton-insoluble fraction after micrococcal nuclease treatment (Figure. 1B) [8]. 
To assess whether DNA replication inhibition could change the solubility of human Claspin as described in Xenopus extracts [6], we followed its presence in Triton soluble and insoluble fractions of cells mock treated or treated with HU. We did not detect any changes in human Claspin solubility in response to DNA replication arrest by HU (Figure 1D). Thus, it seems that chromatin association of the human claspin is not modulated by DNA replication arrest in contrast to Xenopus Claspin.

\section{A fraction of human Claspin immunoprecipitates with ATR and PCNA}

It has been suggested that human Claspin could play a role similar to Xenopus Claspin in regulating the Chk1-dependent DNA damage checkpoint. Claspin has been shown to weakly interact with Chk1 after DNA damage [4] and to be required for ATR-dependent Chk1 phosphorylation in response to DNA damage [9]. In response to DNA replication arrest, a phosphorylated form of Claspin associates with the catalytic domain of Chk1, allowing Chk1 recognizion and therefore phosphorylation by ATR [5]. Surprisingly in xenopus extract, ATR was not been described to associate with Claspin.

We thus investigated whether human Claspin interacts not only with Chk1 as previously shown or also with chk1's direct activator namely ATR. ATR is a member of the PI3-like kinase family with ATM and DNA-PK, and is mainly involved in the DNA replication checkpoint response [10]. ATR phosphorylates Chk1 in vitro and in vivo on at least two SQ residues (ser317 and ser 345) in response to HU or UV treatment [1]. ATR was immunoprepicipated from Hela cell lysates from respectively mock treated, treated with HU or treated with BL. By western blot, both ATR and human Claspin were present in whole cell extracts. While ATR was 
immunoprecipitated under all conditions (Figure. 2A upper panel), associated human Claspin was only seen in proliferating cells and this interaction was dramatically reduced in response to DNA damage (Figure. 2A lower panel). Thus, human Claspin and ATR interactions exist in proliferating cells without detectable activation of the DNA structure checkpoint. Although reciprocal immunoprecipitation was not possible due to lack of immunoprecipitating claspin antibodies, this association further supports the role of adaptator/template protein for Claspin in the ATR-dependent pathway. Human Claspin helps to bring together ATR and its substrates such as Chk1 or BRCA1 in order to facilitate their phosphorylation [5] [9].

In Xenopus extracts, it has been shown that Claspin is associated with the chromatin during ongoing DNA replication and seems to relay replication and checkpoint signals [6]. Interestingly, PCNA, the auxiliary sub-unit of DNA polymerase delta during DNA replication [11] [12] has its functions impaired during DNA damage response [23]. We next wanted to determine whether there was a link between PCNA-dependent on going replication and ATR/claspin-dependent signaling pathway. For that, we look in PCNA-associated complexes for the presence of human Claspin in proliferating or HU treated cells. Both human Claspin and PCNA were present in whole crude extracts analysed by immunoblot in cells mock treated or treated with HU. When PCNA was immunoprepicitated with specific monoclonal antibodies, a fraction of human Claspin co-immunoprecipitated with PCNA (Figure. 2B) but not in control immunoprecipitates. Importantly, this interaction was abolished in cells challenged with HU (Figure 2B right panel). Taken together these data show that a fraction of human Claspin is found not only with ATR complex, a checkpoint sensor kinase but also with PCNA, a central replication protein. Futhermore, upon activation of the $\mathrm{S}$ phase checkpoint the PCNA-Claspin and Claspin-ATR interactions are no longer seen. 
Inhibition of PCNA function leads to Chkl activation

If the DNA replication checkpoint monitors PCNA-dependent ongoing DNA replication through a complex including claspin protein, we anticipated that interfering with PCNA function in cells would lead to the activation of the ATR/claspin/Chk1 pathway. In response to DNA replication perturbation, ATR activates Chk1 by phosphorylation on ser317 and ser 345 in a absolute Claspin-dependent manner [4] leading to cell cycle arrest and recovery. We used phospho specific antibodies (serine 317) of Chk1, to monitor its ATR/Claspin-dependent activation in response to HU by indirect immunofluorescence. Cells were treated or not with $2 \mathrm{mM}$ of $\mathrm{HU}$ for 24 hours, fixed and stained for immunofluorescence with anti phospho Ser317Chk1 (Figure 3A). Whereas only basal phosphorylation of Chk1 on Ser317 was detected in asynchronously growing cells, this phosphorylation dramatically increased in response to HU (Figure. 4A) thus validating that we could monitor ATR-dependent Chk1 activation via this approach.

In order to block PCNA function in vivo, we used a mutant form of p21 protein (W49R, F51S, D52A) previously described to no longer bind Cdks while still specifically inhibiting PCNA function by direct interaction [13]. This mutant when overexpressed in cells, blocks the capability of PCNA to interact with DNA polymerases thus inducing a cell cycle arrest [13] and no detectable DNA replication [13]. This epitope-tagged form of p21 mutant (W49R, F51S, D52A) was transiently transfected in Hela cells. Three days after transfection, cells were fixed and stained with anti HA to detect p21 expression and checked for ATR-dependent Chk1 phosphorylation. As shown on figure $4 \mathrm{~B}$, cells that express the HA-p21 mutant were strongly positive for the phospho active form of Chk1. Finally, stable cell lines expressing this p21 
mutant under control of a tetracycline promoter, display under inducible conditions a high level of HA-p21, concomitent with an accumulation of Chk1 phospho-Ser345 and Chk1 shift up (Figure 3C) [4].

Thus, inhibition of PCNA function induces an ATR/Claspin-dependent Chk1 phosphorylation. This result is similar to the observation made in yeast. Indeed, PCNA inhibition in fission yeast by p21 heterologous overexpression leads to strong Chk1-dependent cell cycle arrest [14].

\section{Discussion}

We report here that human Claspin is a chromatin bound protein in normally proliferating cells independently of its interaction with ATR or PCNA. In response to DNA damage, phosphorylated Xenopus Claspin bound to Chk1 and consequently allows its phosphorylation by ATR [15]. We found with others that ATR associates with human Claspin before DNA damage, favoring the hypothesis that this checkpoint protein is an adaptor between ATR and its substrates such as Chk1 or BRCA1 [4] [9].

One of the key issues in understanding the DNA structure checkpoint is how a replication arrest is detected. ATR like Chk1 is required for cell survival, arguing strongly that these kinases are essential for normal cell cycle [16] [17] in absence of detectable DNA damage. Recent work shows also that in human cells, ATR associates during S phase with chromatin, suggesting its involment in monitoring ongoing replication [18]. In proliferating cells, we show here that Claspin is found in complex with ATR and associated with chromatin. Interestingly, as 
observed in Xenopus extracts, Claspin chromatin binding is independent of its association with ATR (Figures 1 and 2) [6].

Human claspin interacts also with PCNA complex (Figure 2). In a normal cell cycle, PCNA is believed to be a sliding clamp that acts as a processivity factor for DNA polymerases $(\delta$ and $\varepsilon$ ) and also interacts with FEN-1 endonuclease during Okasaki fragment processing [19] [12]. Because human Claspin is a constituvely chromatin bound protein that interacts with PCNA in absence of detectable DNA damage, we speculate that a fraction of human Claspin is part of the DNA replication machinery and could participate in the ATR-dependent DNA replication monitoring. A yeast functional homolog of the Claspin protein, namely MRC1, is found associated with a replication machinery element, namely Cdc45 [20], that helps a loading of DNA polymerase/PCNA onto DNA [21]. Furthermore, MRC1 is also involved in the recovery from DNA replication stress through the stabilization of the stalled replication forks (review in [22]) arguying in favor of its localization onto DNA [23]. It would be interesting in the future to determine whether Claspin protein is found in complex with other replication proteins such as Cdc45 in humans.

Thus, it seems that Claspin protein in higher Eukaryotes allow both fork stabilisation and the ATR/Chk1 Checkpoint-dependent pathway to monitor proper ongoing PCNA-dependent replication. According to this proposed model, inhibition of PCNA function by p21 overexpression leads to ATR/Chk1-dependent checkpoint response (Figure3B-C). Recent evidence proposes that xenopus PCNA functions are impaired [23] in response to DNA damage leading to Xenopus Chk1 activation. We could here observe that direct inhibition of PCNA function induces an ATR/Claspin-dependent Chk1 activation. In the future, it would be interesting to elucidate whether a dissociation of human claspin from PCNA complex in 
response to DNA damage could be part of the normal response to allow proper DNA replication recovery after fork stalling.

Acknowledgments: We thank O. Mondesert for provinding HA-p21 construct. We also thank all members of Cell biology laboratory and the Cell cycle Group of Montpellier specially J. Piette D. Fisher, J.M Lemaitre, D. Marioca, E. Danis, J. M Saffin for help and discussions. This work was supported by Association Contre le Cancer (ARC 3494, France) which was awarded to JMB and a grant from ECC 5th frame work (QLK3-CT-2000-01038) to NJL.

\section{Materiels and methods}

\section{Cells and Culture Conditions, synchronization and drugs}

Hela S3, U2OS cells (American Type Culture Collection), Hela S3 Tet on cells (from promega, France) and p21 mutant expressing DLD1 cell lines [13] were cultivated in Dulbecco's modified Eagle's medium (DMEM; Invitrogen.) containing 10\% fetal calf serum, 50 units $/ \mathrm{ml}$ penicillin, and $50 \mu \mathrm{g} / \mathrm{ml}$ streptomycin sulfate. Cells were treated 24 hours with $2 \mathrm{mM}$ of hydroxyurea or 10 hours with bleomycin $(5 \mathrm{mU} / \mathrm{ml})$ (Calbiochem (CA, USA)). For p21 expression experiment, Hela Tet on cells were transiently transfected with a plasmid encoding the HA tagged form of the p21 mutant (W49R, F51S, D52A) [13] by using lipofectamine2000 agent (according to the manufacturer, Invitrogen, UK).

Preparation of Polyclonal Antisera against Human Claspin, immunodetection of phosphoSer317 of Chk1.

A synthetic peptide corresponding to the 20 amino acids of human Claspin protein conjugated to keyhole limpet hemocyanin was used for rabbit immunization. These antibodies are referred to here as CL\#24. The specificity of CL\#24 was tested by Western blotting using cell lysates from 
hela cells in the presence or absence of $10 \mathrm{mg} / \mathrm{ml}$ of immunogenic peptide. The commercially antibodies against Human Claspin were purchased from Abcam, UK. The polyclonal antibodies against ATR were purchased from Affinity bioreagents, INC, UK). Mouse monoclonal Antibodies (PC10) against PCNA was from Santa Cruz biotech, (Santa Cruz, USA). Rabbit polyclonal Anti PRK was from BD (UK). For Chk1 activation and p21 expression, cells were fixed as previously described [24] stained with anti-phosphoser317 of Chk1 (Upstate, UK) and anti HA monoclonal antibody (12CA5) and revealed with anti rabbit alexa555 and anti mouse alexa488 (Molecular probe, Neth) respectively. Chk1 monoclonal antibodies (8408 from Santa Cruz, USA), anti phosphoSer345 Chk1 (Upstate, UK) and anti HA (12CA5) were used for western-blotting $50 \mu \mathrm{g}$ of DLD1 extracts.

\section{Preparation of cells extracts, immunoprecipitation and Western Blot Analysis}

Cell fractionation was performed as previously described [8]. Each fraction was separated on SDS-PAGE, gel and western blotted for Claspin, PRK and MCM2. For the immunoprecipitation experiment, cells were lysed as previously described ([26]. The concentration of proteins was determined using the bicinchoninic acid protein assay kit (Pierce, USA) with bovine serum albumin as standard. Proteins were immunoprecipitated overnight with specific antibodies and 1 mg of total proteins. Atfer washes immunoprecipitates or whole proteins from cell lysates $(100 \mu \mathrm{g})$ were separated by $7.5 \%$ SDS-PAGE and electrophoretically transferred to Nitrocellulose. Membranes were blocked in Tris-buffered saline $(25 \mathrm{mM}$ Tris- $\mathrm{HCl}, \mathrm{pH} 7.5$, and $137 \mathrm{mM} \mathrm{NaCl}$ ) containing $5 \%$ nonfat dry milk and $0.2 \%$ Tween 20 . The blots were then incubated with CLN\#24 (1:300) or anti ATR (1:200) or anti PCNA (1:200) or anti Claspin (1:250) in blocking solution for $14 \mathrm{~h}$ at $4{ }^{\circ} \mathrm{C}$. After washing in Tris-buffered saline and $0.2 \%$ Tween 20, blots were incubated with horseradish peroxidase-conjugated goat anti-rabbit IgG (1:3000) or horseradish peroxidase-conjugated protein AG (1:5000) in blocking solution for $1 \mathrm{~h}$ and revealed with ECL.

\section{References}

1. Zhao, H., and Piwnica-Worms, H. (2001). ATR-mediated checkpoint pathways regulate phosphorylation and activation of human Chk1. Mol Cell Biol 21, 4129-39. 
2. Melchionna, R., Chen, X. B., Blasina, A., and McGowan, C. H. (2000). Threonine 68 is required for radiation-induced phosphorylation and activation of Cds1. Nat Cell Biol 2, $762-5$.

3. Kumagai, A., and Dunphy, W. G. (2000). Claspin, a novel protein required for the activation of Chk1 during a DNA replication checkpoint response in Xenopus egg extracts. Mol Cell 6, 839-49.

4. Chini, C. C., and Chen, J. (2003). Human claspin is required for replication checkpoint control. J Biol Chem 278, 30057-62.

5. Jeong, S. Y., Kumagai, A., Lee, J., and Dunphy, W. G. (2003). Phosphorylated claspin interacts with a phosphate-binding site in the kinase domain of Chk1 during ATRmediated activation. $J$ Biol Chem 278, 46782-8.

6. Lee, J., Kumagai, A., and Dunphy, W. G. (2003). Claspin, a Chk1-regulatory protein, monitors DNA replication on chromatin independently of RPA, ATR, and Rad17. Mol Cell 11, 329-40.

7. Ouyang, B., Li, W., Pan, H., Meadows, J., Hoffmann, I., and Dai, W. (1999). The physical association and phosphorylation of $\mathrm{Cdc} 25 \mathrm{C}$ protein phosphatase by Prk. Oncogene 18, 6029-36.

8. Todorov, I. T., Attaran, A., and Kearsey, S. E. (1995). BM28, a human member of the MCM2-3-5 family, is displaced from chromatin during DNA replication. J Cell Biol 129, 1433-45.

9. Lin, S. Y., Li, K., Stewart, G. S., and Elledge, S. J. (2004). Human Claspin works with BRCA1 to both positively and negatively regulate cell proliferation. Proc Natl Acad Sci U S A 101, 6484-9.

10. Shiloh, Y. (2001). ATM and ATR: networking cellular responses to DNA damage. Curr Opin Genet Dev 11, 71-7.

11. Gomes, X. V., and Burgers, P. M. (2000). Two modes of FEN1 binding to PCNA regulated by DNA. Embo J 19, 3811-21.

12. Jonsson, Z. O., Hindges, R., and Hubscher, U. (1998). Regulation of DNA replication and repair proteins through interaction with the front side of proliferating cell nuclear antigen. Embo J 17, 2412-25.

13. Cayrol, C., Knibiehler, M., and Ducommun, B. (1998). p21 binding to PCNA causes G1 and G2 cell cycle arrest in p53-deficient cells. Oncogene 16, 311-20.

14. Tournier, S., Leroy, D., Goubin, F., Ducommun, B., and Hyams, J. S. (1996). Heterologous expression of the human cyclin-dependent kinase inhibitor p21Cip1 in the fission yeast, Schizosaccharomyces pombe reveals a role for PCNA in the chk1+ cell cycle checkpoint pathway. Mol Biol Cell 7, 651-62.

15. Kumagai, A., and Dunphy, W. G. (2003). Repeated phosphopeptide motifs in Claspin mediate the regulated binding of Chk1. Nat Cell Biol 5, 161-5.

16. Brown, E. J., and Baltimore, D. (2000). ATR disruption leads to chromosomal fragmentation and early embryonic lethality. Genes Dev 14, 397-402.

17. Liu, Q., Guntuku, S., Cui, X. S., Matsuoka, S., Cortez, D., Tamai, K., Luo, G., CarattiniRivera, S., DeMayo, F., Bradley, A., Donehower, L. A., and Elledge, S. J. (2000). Chk1 is an essential kinase that is regulated by Atr and required for the G(2)/M DNA damage checkpoint. Genes Dev 14, 1448-59.

18. Dart, D. A., Adams, K. E., Akerman, I., and Lakin, N. D. (2004). Recruitment of the cell cycle checkpoint kinase ATR to chromatin during S-phase. J Biol Chem 279, 16433-40. 
19. Warbrick, E. (1998). PCNA binding through a conserved motif. Bioessays 20, 195-9.

20. Katou, Y., Kanoh, Y., Bando, M., Noguchi, H., Tanaka, H., Ashikari, T., Sugimoto, K., and Shirahige, K. (2003). S-phase checkpoint proteins Tof1 and Mrc1 form a stable replication-pausing complex. Nature 424, 1078-83.

21. Bell, S. P., and Dutta, A. (2002). DNA replication in eukaryotic cells. Annu Rev Biochem 71, 333-74.

22. Nyberg, K. A., Michelson, R. J., Putnam, C. W., and Weinert, T. A. (2002). Toward maintaining the genome: DNA damage and replication checkpoints. Annu Rev Genet 36, 617-56.

23. Stokes, M. P., and Michael, W. M. (2003). DNA damage-induced replication arrest in Xenopus egg extracts. J Cell Biol 163, 245-55.

24. Turowski, P., Franckhauser, C., Morris, M. C., Vaglio, P., Fernandez, A., and Lamb, N. J. (2003). Functional cdc25C dual-specificity phosphatase is required for S-phase entry in human cells. Mol Biol Cell 14, 2984-98.

25. Kitzmann, M., Vandromme, M., Schaeffer, V., Carnac, G., Labbe, J. C., Lamb, N., and Fernandez, A. (1999). cdk1- and cdk2-mediated phosphorylation of MyoD Ser200 in growing C2 myoblasts: role in modulating MyoD half-life and myogenic activity. Mol Cell Biol 19, 3167-76.

26. Bao, S., Tibbetts, R. S., Brumbaugh, K. M., Fang, Y., Richardson, D. A., Ali, A., Chen, S. M., Abraham, R. T., and Wang, X. F. (2001). ATR/ATM-mediated phosphorylation of human Rad17 is required for genotoxic stress responses. Nature 411, 969-74.

Figures legends:

Figure 1: chromatin binding of human Claspin

(A) Characterization of the polyclonal antibodies against human Claspin. Hela cells were treated or not with HU ( $2 \mathrm{mM}$ from Sigma), Human Claspin is detected as a major single band around $170 \mathrm{kDa}$ with both CLN\#24 (left panel) and commercially available antibodies (from Abcam, UK) (right panel). Signal is removed in presence of immunogenic peptide $(10 \mu \mathrm{g} / \mathrm{ml})$ (middle panel) (B) Extractability of human Claspin protein. Subcellular fractionation was performed as described. Total (T), Triton soluble (S) and Triton insoluble fractions (I) were separed on SDS PAGE and western blotted with anti Claspin, anti PRK and anti MCM2. (C) human claspin solubilization by DNAse treatment. Previously described Triton insoluble fraction (I) were treated with micrococcal nuclease as described by manufacturer (USB, UK), then washed and separared by SDS PAGE. Human claspin was revealed by immunoblot. (D) Extractability of human Claspin in DNA damage treated cells. Cells were treated (HU) or not (AS) with $2 \mathrm{mM} \mathrm{HU}$, and cell fractionation experiment was performed as described. T, S and I fractions were separated and immunoblotted with anti-Claspin. PCNA was used as control.

Figure 2: Association of human Claspin with ATR and PCNA. (A) ATR immunoprecipitates contain Claspin protein. Cells were treated or not (AS) with HU or Bleomycin (B1), lysed and ATR was immunoprecipitated then western blotted for ATR and for a presence of human Claspin. (B) PCNA immunoprecipitates contain Claspin. 
Cells were treated (HU) or not (AS) with HU, lysed, PCNA was immunoprecipated and revealed by western blotting. Human Claspin was observed with PCNA in proliferative cell extracts. Anti HA monoclonal antibody was used as negative control for PCNA immunoprecipitation (con).

Figure 3: Inhibition of PCNA leads to Chk1 phosphorylation. (A) U2OS cells were treated or not with $2 \mathrm{mM}$ of $\mathrm{HU}$, fixed and stained with phospho-Ser317 Chk1 antibodies in red or with Hoescht for DNA in blue. (B) p21 mutant (W49R, F51S, D52A) overexpression induces Chk1 phosphorylation on Ser317 in vivo. Hela Tet on cells were transiently tranfected with p21 expressing vector. 24 hours after transfection, deoxycycline $(10 \mu \mathrm{g} / \mathrm{ml})$ was added for 48 more hours. Cells were fixed and stained with anti HA antibody for p21 expression (in green), with phospho-Ser317 Chk1 antibodies in red, and with Hoechst for DNA in blue. (C) DLD1 cell lines expressing p21 mutant were left in presence or in absence of doxycline for 72 hours. Cell extracts were separated on SDSPAGE and westernblotted with anti Chk1, anti phospho-Ser345 Chk1 or with anti HA. 